\title{
Treatment of neovascular age-related macular degeneration in patients with diabetes
}

\author{
Michael Cummings' \\ José Cunha-Vaz ${ }^{2}$ \\ 'Academic Department of Diabetes \\ and Endocrinology, Queen \\ Alexandra Hospital, Portsmouth, \\ UK; ${ }^{2}$ Department of Ophthalmology, \\ University Hospital of Coimbra, \\ Centre of Ophthalmology, Institute \\ of Biomedical Research on Light and \\ Image, Faculty of Medicine, University \\ of Coimbra, and Association for \\ Innovation and Biomedical Research \\ on Light and Image, Coimbra, Portugal
}

\begin{abstract}
The number of patients with type 2 diabetes continues to rise; an anticipated 300 million people will be affected by 2025 . The immense social and economic burden of the condition is exacerbated by the initial asymptomatic nature of type 2 diabetes, resulting in a high prevalence of micro- and macrovascular complications at presentation. Diabetic retinopathy, one of the potential microvascular complications associated with diabetes, and neovascular age-related macular degeneration (AMD) are the two most frequent retinal degenerative diseases, and are responsible for the majority of blindness due to retinal disease. Both conditions predominantly affect the central macula, and are associated with the presence of retinal edema and an aggressive inflammatory repair process that accelerates disease progression. The associated retinal edema and the inflammatory repair process are directly involved in the breakdown of the blood-retinal barrier (BRB). Yet, the underlying alterations to the BRB caused by the diseases are very different. The coexistence of the two conditions appears to be relatively uncommon, suggesting that diabetes may even protect patients from developing neovascular AMD. However, it is thought that the inflammatory repair responses associated with diabetic retinopathy and neovascular AMD may be cumulative and, in patients affected by both, could result in chronic diffuse cystoid edema. Treatment considerations in such patients should, therefore, include the role of retinal edema and the increased susceptibility of patients with diabetes to potential systemic side effects associated with agents administered repeatedly for neovascular AMD treatment.
\end{abstract}

Keywords: diabetes, diabetic retinopathy, neovascular age-related macular degeneration, retina, edema

\section{Introduction}

In 1999, the World Health Organization (WHO) reclassified the biochemical and etiological definitions of diabetes mellitus (see Table 1) (WHO 1999). These changes were helpful in better identifying individuals associated with progressively greater risk of developing the pathognomonic microvascular and macrovascular complications associated with diabetes.

\section{Diabetes prevalence}

Type 2 diabetes represents more than $85 \%$ of all cases of diabetes. The WHO estimated that worldwide, 150 million people over the age of 20 years had the condition in 2000, a number set to rise to 300 million people by 2025 (King et al 1998). The prevalence varies enormously between countries, depending upon numerous genetic and environmental factors. However, the greatest burden appears to be in India, China, the US, Pakistan, Indonesia, the Russian Federation, Mexico, and Brazil, which collectively account for more than half of all cases globally (King et al 1998). Expressed as age-specific prevalence, less than $10 \%$ of the European population under 60 years old have type 2 diabetes, yet this increases to $10 \%-20 \%$ in those aged $60-79$ years (DECODE 2003). 
Table I WHO classification of diabetes mellitus (WHO 1999)

*Fasting venous plasma $\geq 7.0 \mathrm{mmol} / \mathrm{L}$

*Random venous plasma $\geq 11.1 \mathrm{mmol} / \mathrm{L}$

Type I (beta-cell destruction, usually absolute insulin deficiency)

IA Autoimmune

IB Idiopathic

Type 2 (range from predominant insulin resistance to predominantly secretory defect \pm insulin resistance)

\section{Other specific types}

Genetic defects of beta cell function or insulin action

Diseases of the exocrine pancreas

Endocrinopathies

Drug/chemical induced

Infections

Rare immune-mediated forms

Other genetic disorders associated with diabetes

\section{Gestational diabetes}

*One abnormal blood glucose measurement is sufficient in the presence of symptoms, but for the asymptomatic person at least one additional plasma/blood glucose test result with a value in the diabetic range is essential.

Worryingly, the incidence of pediatric type 2 diabetes is rising and, exceeds 4\% in American Indians aged between 15-19 years (Dabelea et al 1998). The asymptomatic nature of this condition, common until complications emerge, increases the burden of diabetes as, for example, up to one third of patients may have retinopathy at diagnosis. There is a resultant need for more widespread, efficient screening to enable earlier capture of new cases, but there is no universal agreement as to how this can be best achieved.

In contrast to type 2 diabetes, the relatively abrupt onset of type 1 diabetes usually negates the likelihood of diabetic complications at diagnosis (less than $2 \%$ of patients have retinopathy at presentation). It is estimated that 10-20 million individuals suffer from this condition globally (Rewers et al 1988). Type 1 diabetes peaks in patients under the age of 15 years (with prevalence of up to $0.3 \%$ ) and has dramatic geographic variability. A child in Finland, for example, is 400 times more likely to develop type 1 diabetes than a child in China (Steck et al 2004).

\section{The burden of diabetes}

The implications of diabetes are immense at an individual, caregiver and societal level. When considering some of the potential burdens faced by individuals with diabetes, as summarized in Table 2, it is not surprising that these patients are prone to states of depression and anxiety (Gavard et al 1993).

The Costs of Diabetes in Europe-2 (CODE-2) Study (Baxter et al 2000) and T2ARDIS Study (GSK 2000) perhaps provide the most robust data on the economic burden associated with diabetes across Europe. These studies have identified that the healthcare costs associated with patients with diabetes are more than twice those of age-matched individuals without diabetes. Using a UK-based model, this lifetime cost equates to $£ 33,000$ for male patients and $£ 43,000$ for female patients, compared with $£ 15,000$ for men and $£ 22,000$ for women without diabetes (Bagust et al 2001).

CODE-2 also demonstrated that the costs of those patients with microvascular complications, such as retinopathy, are nearly twice those associated with patients without complications. These calculations underestimate the true cost burden associated with diabetes as they fail to take into account absence from work, early retirement or premature death due to the condition. Evidence from studies in both type 1 (DCCT 1996) and type 2 (UKPDS 2000) diabetes suggest that better glycemic control is cost-effective in reducing diabetes-associated morbidity and mortality. Systematic screening for diabetic complications such as retinopathy has also been shown to be a cost-effective intervention (James et al 2000).

\section{The pathogenesis of diabetes Type I process}

Type 1 diabetes indicates the presence of underlying pancreatic beta-cell destruction with a progressive decline in insulin production and secretion. Exogenous insulin administration is required for survival to prevent the development of

Table 2 Examples of the individual burden of diabetes

\begin{tabular}{ll}
\hline Lifestyle & $\begin{array}{l}\text { Dietary, exercise, and changes to other factors associated } \\
\text { with diabetes } \\
\text { Monitoring and management of diabetes } \\
\text { Westrictions for sports and other leisure activities } \\
\text { Vehicle driving limitations }\end{array}$ \\
& $\begin{array}{l}\text { Restrictions on occupations } \\
\text { Implications at work for monitoring/managing diabetes }\end{array}$ \\
Clinical & $\begin{array}{l}\text { Need for regular health checks/blood tests } \\
\text { The development (or fear of development) of diabetic } \\
\text { complications and their implications }\end{array}$ \\
& $\begin{array}{l}\text { Greater likelihood of hospital admission } \\
\text { Often the need for regular medication with risks of side } \\
\text { effects } \\
\text { Increased risk of other conditions associated with } \\
\text { diabetes }\end{array}$ \\
Costs of treatment (in non-state health subsidized \\
societies) \\
Loading of health-related insurances \\
Loss of income through diabetes-related illness
\end{tabular}


ketoacidosis, coma and death. Type $1 \mathrm{~A}$ diabetes is thought to arise from a chronic autoimmune destruction of the pancreatic beta cells, usually initiated by exposure of genetically susceptible individuals to environmental agents. This event is usually a cell-mediated immune process and is characterized by the presence of islet cell, insulin or glutamic acid decarboxylase (GAD) autoantibodies. Genetic influences include the presence of susceptibility genes, such as in the human leukocyte antigen (HLA) region on chromosome 6 p21, which accounts for nearly half of familial clustering. Potential environmental factors have implicated viruses (eg, herpes virus, mumps, rubella, and retroviruses), perinatal factors, dietary factors, and exposure to toxins (Steck and Rewers 2004).

Type 1B diabetes refers to a heterogeneous group of individuals who show no evidence of autoimmune destruction of pancreatic beta cells; they account for up to $40 \%$ of patients with type 1 diabetes (Urakami et al 2002).

\section{Type 2 process}

The process underling type 2 diabetes is insulin resistance and defective insulin secretion, either of which predominates. Type 2 diabetes is heterogeneous and has many distinct causes. However, most patients are obese with concomitant insulin resistance and fulfill the classification of the metabolic syndrome (see Table 3) (Alberti et al 1998).

\section{Diabetic complications Macrovascular}

The major abnormality present in diabetes is atherosclerosis (Kannel et al 1979), usually preceded by endothelial dysfunction. Much debate surrounds whether this is an exaggeration of the process seen in non-diabetic subjects, or whether there are specific features pathognomonic of diabetes. Overall, diabetes seems to be associated with an increase in amount, extent, and distribution of atherosclerosis leading to susceptibility to ischemic heart

Table 3 The metabolic syndrome (modified WHO definition) (Alberti et al 1998)

- Fasting plasma glucose $\geq 6.1 \mathrm{mmol} / \mathrm{L}$ or hyperinsulinemia (upper quartile of the non-diabetic range), and

At least 2 of the following:

- Abdominal obesity: $\mathrm{BMI} \geq 30 \mathrm{~kg} / \mathrm{m}^{2}$, waist-hip ratio $>0.90$ or waist circumference $\geq 94 \mathrm{~cm}$

- Dyslipidemia: serum triglycerides $\geq 1.7 \mathrm{mmol} / \mathrm{L}$ or $\mathrm{HDL}$ cholesterol $<0.4 \mathrm{mmol} / \mathrm{L}$

- Hypertension: blood pressure $\geq 140 / 90 \mathrm{mmHg}$ or medication

Abbreviations: BMI, body mass index; HDL, high density lipoprotein. disease, cerebrovascular and peripheral vascular disease and erectile dysfunction. The association between glycemic control and macrovascular disease is less pronounced compared with other vascular risk markers such as smoking, hypertension and dyslipidemia.

\section{Microvascular}

Microvascular complications consist of pathological changes that mainly affect the function of the retina, kidney and neurological system, and a much more established link to the duration and severity of hyperglycemia (DCCT 1996; UKPDS 2000). The role of hyperglycemia plays in inducing these complications at a cellular level is now better understood. Within the retina itself, the classical finding is that of basement membrane thickening, which is associated with leakage and a loss of negative charge. Altered pericyte function, increased blood viscosity and altered fibrinolytic activity may lead to capillary occlusion with tissue hypoxia and, ultimately, new vessel formation. In addition to hyperglycemia, a number of other risk factors are associated with development or worsening of retinopathy, including hypertension, nephropathy, pregnancy, alcohol, smoking and ethnic origin (Shotliff et al 2005). The classification of different forms of diabetic retinopathy is now well established (see Table 4).

\section{Distinction between diabetic retinopathy and wet $A M D$ Diabetic retinopathy}

Diabetic retinopathy is a characteristic group of lesions found in the retina of individuals who have had diabetes for a number of years. Successful management of glycemia reduces the risk of developing, and slows the progression of, retinopathy. However, in many cases, diabetic retinopathy can result in progressive visual loss in well-controlled patients. It is important to realize that the natural history of diabetic retinopathy does not follow a standard timetable of progression; contrastingly, it has a rather variable course. Factors that most influence the evolution of the disease are the type and duration of diabetes, increased blood pressure and pregnancy. In the pre-clinical stage, alterations preceding ophthalmoscopic changes have been demonstrated by a variety of more sensitive methods of examination and by histological examination (Cunha-Vaz and Bernardes 2005).

Diabetic retinopathy is essentially a microangiopathy: its initial pathologic processes include endothelial proliferation in the capillaries and venules, as well as endothelial swelling and apoptosis in the smallest arteriolar branchings. 
Table 4 Classification of diabetic retinopathy

\section{Background retinopathy}

Capillary microaneurysms ('dots')

Intraretinal hemorrhages ('blots')

Hard exudates (lipid exudates)

Pre-proliferative retinopathy

Soft exudates (cotton wool spots, infarcts)

Intra-retinal microvascular abnormalities (IRMAs)

Venous abnormalities (beading, looping, and re-duplication)

Proliferative retinopathy

New vessels on the disc or within one disc diameter (NVD)

New vessels elsewhere (NVE)

Rubeosis iridis ( \pm neovascular glaucoma)

Maculopathy

Edematous

Exudative

Ischemic

Any combination of above

Pericyte damage is also present, but its distribution is irregular and is only made more apparent because the affected cells are encased in basement membrane and thus take longer than endothelial cells to be replaced when damaged.

Fluorescein angiography confirmed the histopathological findings of diabetic retinal disease in a dynamic manner. It was the first technique to document the abnormal leakage of fluorescein through the walls of the retinal vessels, demonstrating a breakdown of the blood-retinal barrier (BRB).

Vitreous fluorophotometry has been used to quantify the alteration of the BRB (Cunha-Vaz et al 1975). The method has confirmed that alteration of the BRB.

When alterations of the fundus are detected by ophthalmoscopy, it is said that non-proliferative diabetic retinopathy is present. The clinical features of non-proliferative diabetic retinopathy are: microaneurysms, intra-retinal hemorrhages, hard exudates and variable degrees of retinal edema. Retinal microaneurysms are usually the first ophthalmoscopic sign of diabetic retinopathy. New microaneurysms are constantly being formed while older ones become occluded and the rate of formation of new microaneurysms may be the most relevant marker of disease progression. Retinal edema is a frequent finding that leads to distortion of visual images and may cause a significantly decreased visual acuity, even in the relatively early stages of the retinopathy.

Stereoscopic fundus photography and slit-lamp microscopy have played an important role by demonstrating changes in retinal volume in the macular area, but findings are dependent on the observer's experience, and the results do not offer a reproducible measurement of the volume change.
Two new techniques that make objective measurements of changes in retinal thickness have recently become available: optical coherence tomography (OCT) and the retinal thickness analyzer (RTA) (Zeimer et al 1989; Puliafito et al 1995).

It is now possible to measure changes in retinal thickness and identify macular edema using non-invasive instrumentation in a clinical setting. Diabetic macular edema may now be identified by its type and distribution, evolution, pathophysiology, and by the degree of involvement of the central macular area (Cunha-Vaz 2006) (see Table 5).

Pre-proliferative diabetic retinopathy is an intermediate stage between non-proliferative and proliferative retinopathy. Widespread and increasing capillary closure is the fundamental feature of the pre-proliferative retinopathy stage. There are also soft exudates, venous beading and intraretinal microvascular abnormalities (IRMA). In addition, proliferative retinopathy is characterized by the presence of new vessels that may arise from the optic disk or from the retina periphery. These new vessels initially lie in the plane of the retina, but quickly pierce the internal limiting membrane and become pre-retinal, forming adhesions with the overlying vitreous. The presence of the new vessels leads to retraction of the vitreous. This pulling effect causes the progressive complications associated with retinal neovascularization, such as vitreous hemorrhage, progressive visual distortion and traction retinal detachment. Neovascularization always develops in eyes that show generalized and widespread capillary non-perfusion, again confirming that it is a direct consequence of generalized ischemia.

\section{Neovascular age-related macular degeneration}

Age-related Macular Degeneration (AMD) occurs in two main forms: early (ie, dry) AMD and late (ie, neovascular/ exudative/wet) AMD (Ambati et al 2003).

Table 5 Diabetic macular edema - characterization

\begin{tabular}{lll}
\hline Characteristics & Focal & Diffuse \\
\hline Chronicity & Not chronic & Chronic \\
Foveal involvement & Fovea preserved & Partial foveal involvement \\
BRB (inner) & No leakage & Open BRB \\
BRB (outer [RPE]) & No RPE dysfunction & RPE dysfunction \\
OCT cysts & None & OCT cysts present \\
Traction & None & Traction present \\
Ischemia (FAZ) & None & Ischemia present \\
HbAlc & HbAlc $<8 \%$ & HbAlc $\geq 8 \%$ \\
Blood pressure (BP) & BP $\leq 130 / 80 \mathrm{mmHg}$ & BP $>\mid 30 / 80 \mathrm{mmHg}$ \\
\hline
\end{tabular}

Abbreviations: BRB, blood-retinal barrier; RPE, retinal pigment epithelium; FAZ, foveal vascular zone; $\mathrm{HbAlc}$, glycated hemoglobin; OCT, optical coherence tomography. 
The accepted clinical hallmark of dry AMD is the appearance of drusen: localized deposits lying between the basement membrane of the retinal pigment epithelium (RPE) and Bruch's membrane. Clinically, drusen are classified morphologically as either hard or soft. Soft drusen are generally larger and have indistinct edges, and have a tendency to become confluent. Typically, drusen are clustered in the central macula, where the pathological abnormalities in AMD are most pronounced. At various stages, the RPE, photoreceptors and choroidal vasculature are involved.

Geographic atrophy is characterized by the presence of confluent areas of RPE cell damage accompanied by overlying photoreceptor atrophy. Geographic atrophy can develop following fading of drusen, in areas of RPE attenuation, following flattening of an RPE detachment, or after involution of choroidal neovascularization (CNV). The visible atrophy is usually accompanied by an atrophic underlying choriocapillaris. Both geographic atrophy and CNV tend to develop in patients with large drusen and pigmentary changes. Patients with bilateral atrophy can develop CNV at a rate of $2 \%-4 \%$ over a 2 -year period.

The development of CNV (ie the growth of new blood vessels from the choroids) is the hallmark of neovascular (or exudative/wet) AMD. In AMD, the new vessels can remain beneath the RPE, or may breach the RPE and enter the retina space. The earliest signs of $\mathrm{CNV}$ are retinal edema and visual distortions.

The fluorescein angiographic leakage patterns of CNV are classified as either classic or occult. The former refers to discrete areas that hyperfluoresce early in the examination and continue to exhibit progressive leakage with increasing intensity and extent. Occult CNV refers either to a fibrovascular RPE detachment with irregular elevation of the RPE, with stippled hyperfluorescence and late leakage, or to late leakage of undetermined origin.

Retinal edema is particularly well demonstrated by OCT examination, and is indicated by an increase in thickness of the retina. It is one of the most widely accepted criteria for treatment and re-treatment of neovascular AMD. There are two special subtypes of wet AMD that are better identified with indocyanine green angiography (ICG): chorioretinal anastomoses and polypoidal-choroidal neovascularization (Slakter et al 2000; Yannuzzi et al 1990). The two exhibit different patterns of disease progression and have different prognoses. The Disciform Scar Represents End-Stage Disease.

Diabetic retinopathy and neovascular AMD are the two most frequent retinal degenerative diseases and are responsible for the majority of cases of blindness due to retinal disease. The two conditions predominantly affect the central macula; their development is associated with the presence of retinal edema and an aggressive inflammatory repair process that accelerates disease progression. Both the retinal edema and the inflammatory repair process are directly associated with breakdown of the BRB. However, the underlying alterations to the BRB are very different in these two diseases. Diabetic retinopathy is, initially, a disease of the inner layers of the retina, beginning with an alteration of the inner BRB, located in the retinal endothelial cells. Contrastingly, neovascular AMD initially affects the outer retinal layers and starts at the chorioretinal interface (with an alteration of the outer BRB), located in the retinal pigment epithelium, which then allows invasion of the retina by neovessels located in the choriocapillaries.

\section{Treatment of neovascular AMD in patients with diabetes}

The population-based Framingham Eye Study, in addition to a number of other case-controlled studies, failed to find an association between macular degeneration and diabetes mellitus. Data from the Beaver Dam Study (Klein et al 1992) suggest that diabetes is not related to macular drusen or geographic atrophy. The only association demonstrated was that between exudative macular degeneration and diabetes in men aged 75 years or older.

In a different study (Zylbermann et al 1997), the prevalence of neovascular AMD in patients with diabetic retinopathy was only $0.86 \%$, which is lower than the prevalence in the general population. Furthermore, the investigators reported that patients treated with laser photocoagulation for diabetic retinopathy are less prone to the development of neovascular AMD.

More recently, data were reported from a 10-year followup study of newly diagnosed patients with type 2 diabetes in Finland (Voutilainen-Kaunisto et al 2000). The study demonstrated that the incidence of neovascular AMD over time was possibly lower in the patients with diabetes compared with control subjects.

Furthermore, the diabetic retinopathy fundus photography screening program in progress in the central region of Portugal has detected an incidence of diabetic retinopathy in $2.6 \%$ of patients with type 2 diabetes, but an incidence of only $0.4 \%$ of all types of AMD (and $0.05 \%$ of neovascular AMD). These values are clearly lower than the values reported for the general population, even taking into consideration the age of the patients examined. 
Therefore, it may be concluded from both literature and clinical experience that diabetes may offer some degree of protection from the development of neovascular AMD.

\section{Current opinion in neovascular AMD treatment}

Although laser photocoagulation was the only treatment available for many years, it is now only accepted for treatment of patients with well defined, extrafoveally located CNV. Photodynamic therapy (PDT), which was approved in 2000 predominantly for the treatment of $\mathrm{CNV}$, has been shown to prevent progression of vision loss when compared with controls, but the main result is temporary stabilization of visual acuity loss.

Combining PDT with anti-inflammatory drugs (such as triamcinolone or other steroids) has offered improved visual outcomes through a reduction of the inflammation caused by PDT. However, the use of steroids can induce several complications. In an attempt to deal with these problems, a variety of sustained-release devices have been introduced to modulate drug delivery to the eye.

Although the current evidence base is inconclusive, it is expected that the inflammatory repair responses present in both diabetic retinopathy and neovascular AMD may be cumulative and may be compounded when wet AMD is present in eyes of patients with diabetic retinal disease.

Vascular endothelial growth factor (VEGF) inhibitors are now being used for the treatment of eyes with neovascular AMD, and have shown a significant degree of success. Treatment with these anti-VEGF agents has even demonstrated that a degree of recovery of visual function, followed by stabilization of such recovery, is possible. Pegaptanib sodium (Macugen $^{\circledR}$, [OSI] Eyetech Pharmaceuticals Inc, New York NY, USA), a selective inhibitor of VEGF, has shown efficacy in trials including all angiographic types of neovascular AMD (Gragoudas et al 2004). The series of trials, together termed VISION, were specifically designed not to differentiate patients on angiographic indications, but rather with the intention of validating the hypothesis that selective inhibition of VEGF could provide a safe and effective treatment for patients of all subtypes of neovascular AMD.

Ranibizumab (Lucentis ${ }^{\circledR}$, Genentech Inc, South San Francisco, CA, USA) is a non-selective anti-VEGF agent and approaches inhibition of VEGF in more general terms. In clinical trials (Brown et al 2006; Rosenfeld et al 2006), the agent has demonstrated that it not only stabilizes the progression of neovascular AMD, but can also lead to an improvement in vision in patients with specific subtypes of the disease. A series of anecdotal reports have confirmed these findings with bevacizumab (Avastin ${ }^{\circledR}$, Genentech Inc, South San Francisco, CA, USA), a drug of the same class, but which is indicated for colorectal cancer and has no license for use in the eye, and has not been submitted for clinical trial evaluation.

Addressing CNV with a non-selective VEGF inhibition, as is the approach of ranibizumab, has demonstrated better visual outcomes. These data may suggest that selective inhibition of VEGF is an important component of combating the disease process, but that there are also other factors present that require treatment. This argument favors combination approaches for treatment of neovascular AMD.

\section{Treatment of neovascular AMD in patients with diabetes}

There are at least two important factors to consider when treating neovascular AMD in patients with diabetes: the role of retinal edema in the modulation of treatment and the relevance of associated side effects of the treatment.

Evaluation of treatment and re-treatment timing is now very much associated with the degree and evolution of retinal edema detected by OCT. Retinal edema is the most frequent alteration in the diabetic retina and its presence may delay recovery and drying of the retina immediately after treatment for neovascular AMD.

Also of note is the consideration of potential side effects that may be induced by repeated treatments with less selective anti-VEGF agents. Any such side effects may be particularly relevant in patients with diabetes, in light of their associated micro- and macrovascular complications.

\section{Conclusions}

Current evidence demonstrates that the coexistence of diabetic retinopathy and neovascular AMD is uncommon, potentially suggesting that diabetes may offer a protective role against the development of neovascular AMD. This interesting hypothesis may be explained by the fact that each disease is initiated by selective involvement of one of the two components of the BRB. The early stages of diabetic retinopathy begin with alterations of the inner BRB, whereas AMD involves the outer BRB. There is some evidence in the literature indicating that, in the case of diabetic retinal edema, there is signaling from the damaged inner BRB that induces up-regulation of the transport function of the RPE (outer BRB) (Sander et al 2001). As a result, diabetic retinal disease may help delay development of the AMD alterations that lead to wet AMD.

In patients with diabetes who require treatment for neovascular AMD, the choice of therapy and timing of treatments and re-treatments (based on degree of retinal edema measured 
by OCT), should take into consideration the association of diabetic retinopathy and the possible presence of chronic diffuse cystoid edema. Another factor that must be considered in patients with diabetes is their potential increased susceptibility to the side effects associated with the drugs administered repeatedly for neovascular AMD treatment.

\section{References}

Alberti KGMM, Zimmet PZ, on behalf of the World Health Organization (WHO). 1998. Definition diagnosis and classification of diabetes mellitus and its complications. Diab Med, 15:539-53.

Ambati J, Ambati BK, Yoo SH, et al. 2003. Age-related macular degeneration: etiology, pathogenesis, and therapeutic strategies. Surv Ophthalmol, 48:257-93.

Bagust A, Hopkinson PK, Maier W, et al. 2001. An economical model of the long-term healthcare burden of type II diabetes. Diabetologia, 44:2140-55.

Baxter H, Bottomley J, Harvey J, et al. 2000. CODE 2 UK: the current costs of type 2 diabetes in the UK. Diab Med, 17(S1):abstract.

Brown DM, Kaiser PK, Michels M, et al. 2006. Ranibizumab versus verteporfin for neovascular age-related macular degeneration. $N$ Engl $J$ Med, 355:1432-44.

Cunha-Vaz JG, Abreu, JRF, Campos AJ, et al. 2006.Early breakdown of the blood-retinal barrier in diabetes. Br J Ophthalmol, 59:649-56.

Cunha-Vaz JG, Bernardes R. 2005. Non-proliferative retinopathy in diabetes type 2. Initial stages and characterization of phenotypes. Progr Retinal Eye Res, 24:355-77.

Cunha-Vaz JG. 1975. Clinical characterization of diabetic macular edema. Int Ophthalmol, 1(4):99-100.

Dabelea D, Hanson RL, Bennett PH, et al. 1998. Increasing evidence of type II diabetes in American Indian children. Diabetologia, 41:904-10.

[DCCT] The Diabetes Control and Complications Trial Research Group. 1996. Lifetime benefits and costs of intensive therapy as practiced in the DCCT. JAMA, 276:1409-15.

[DECODE] Diabetes Epidemiology: Collaborative analysis of Diagnostic criteria in Europe Study Group. 2003 Age- and sex-specific prevalence of diabetes and impaired glucose regulation in 13 European cohorts. Diabetes Care, 26:61-9.

Gavard JA, Lustman PJ, Clouse RE. 1993. Prevalence of depression in adults with diabetes. An epidemiological evaluation. Diabetes Care, 16:1167-78.

Gragoudas ES, Adamis AP, Cunningham Jr ET, et al. 2004. VEGF inhibition study in ocular neovascularization clinical trial group: Pegaptanib for neovascular age-related macular degeneration. $N$ Engl J Med, 351:2805-16.

[GSK] GlaxoSmithKline. 2000. The true costs of type 2 diabetes in the UK - findings from the T2ARDIS and CODE-2. Uxbridge UK: GlaxoSmithKline.

James M, Turner DA, Broadbent DM, et al. 2000. Cost effectiveness of screening for sight threatening diabetic eye disease. BMJ, 320:1627-31.
Kannel WB, McGee DL. 1979. Diabetes and cardiovascular disease. The Framingham Study. JAMA, 241:2035-38.

King H, Aubert RE, Herman WH. 1998. Global burden of diabetes, 1995-2025: prevalence, numerical estimates, and projections. Diabetes Care, 21:1414-31.

Klein R, Klein BE, Moss SE. 1992. Diabetes, hyperglycemia, and agerelated maculopathy. The Beaver Dam Eye Study. Ophthalmology 99:1527-34.

Puliafito CA, Hee MR, Lin CP, et al. 1995: Imaging of macular disease with optical coherence tomography. Ophthalmology, 102:217-229.

Rewers M, LaPorte RE, King H, et al. 1988. Trends in the prevalence and incidence of diabetes; insulin dependent diabetes in childhood. World Health Stat Q, 41:179-89.

Rosenfeld PJ, Brown DM, Heier JS, et al. 2006. Ranibizumab for neovascular age-related macular degeneration. $N$ Engl $J$ Med, 335:1419-31.

Sander B, Larsen M, Moldow B, et al. 2001. Diabetic macular edema: passive and active transport of fluorescein through the blood-retina barrier. Invest Opthalmol Vis Sci, 42:433-8.

Shotliff K, Duncan G. 2005. Diabetes and the eye. In Shaw KM, Cummings MH (eds). Diabetic complications. Chichester UK: John Wiley and Sons. p. 1-19.

Slakter JS, Yannuzzi LA, Schneider U, et al. 2000. Retinal choroidal anastomoses and occult choroidal neovascularization in age-related macular degeneration. Ophthalmology, 107:742-53.

Steck AK, Rewers MJ. 2004. Epidemiology and geography of type 1 diabetes. In DeFronzo RA, Ferrannini E, Keen H, et al. (eds). International textbook of diabetes. Chichester UK: John Wiley and Sons. p. 15-31.

[UKPDS] United Kingdom Prospective Diabetes Study Group. 2000. Cost effectiveness analysis of an intensive blood glucose control policy in patients with type 2 diabetes; economic study analysis alongside randomised controlled trial (UKPDS41) BMJ, 320:1373-8.

Urakami T, Inami I, Morimoto S, et al. 2002. Clinical characteristics of non-immune mediated, idiopathic type 1B diabetes mellitus in Japanese children and adolescents. J Paediatr Endocrinol Metab, 15:283-8.

Voutilainen-Kaunisto RM, Teräsvirta ME, Uusitupa MIJ, et al. 2000. Agerelated macular degeneration in newly diagnosed type 2 diabetic patients and control subjects. Diabetes Care, 23:1672-8.

[WHO] World Health Organization. 1999. Definition, diagnosis and classification of diabetes mellitus and its complications. Report of a WHO Consultation Part 1: Diagnosis and Classification of Diabetes Mellitus. Geneva:WHO.

Yannuzzi LA, Sorenson J, Spaide RF, et al. 1990. Idiopathic polypoidal choroidal vasculopathy (IPCV). Retina, 10:1-8

Zeimer R, Mori MT, Khoobebi B. 1989. Feasibility test of a new method to measure retinal thickness non-invasively. Invest Ophthalmol Vis Sci, 30:2099-105.

Zylbermann R, Landau D, Rozenman Y, et al. 1997. Exudative age-related macular degeneration in patients with diabetic retinopathy and its relation to retinal laser photocoagulation. Eye, 11:872-5. 
